\title{
Journey to Crime Using Dijkstra's Algorithm
}

\author{
Olusina, J.O. ${ }^{1 * *} \&$ Olaleye, J.B. ${ }^{1}$ \\ ${ }^{1}$ Department of Surveying \& Geoinformatics, Faculty of Engineering, University of Lagos, Lagos, Nigeria \\ Corresponding Author: *joolusina1@ yahoo.com
}

\begin{abstract}
This paper describes some benefits of crime mapping in a Geographic Information Systems (G.I.S.) environment. The underlining principle of Journey to Crime was discussed. Crime Spots and Police Stations in the study area were mapped, Shortest-Path, Closest Facility, Service Area and OD (Origin - Destination) Cost Matrix were determined based on Dijkstra's Algorithm. Results show that the distribution of police stations does not correspond with the spread of crime spots.
\end{abstract}

Keywords: Crime Mapping, Geographic Information Systems (G.I.S.), Dijkstra's Algorithm, Policing, Criminal, Shortest-Path.

\subsection{Introduction}

Over the last few years, a new worldwide socio-economical order led to an increasing number on crime rates and raised the need to find new ways to handle information about criminality. To better understand its causes, local, regional and national security authorities turned to new decision support tools such as Geographic Information Systems (GIS) and other information technologies to help them in finding better solutions. The spatial attributes of this phenomena implies quantitative and qualitative variables and to understand its magnitude, it is necessary to capture, analyze and correlate them (Ferreira, João and Martins, 2012).

The advent of easy-to-use GIS applications has allowed crime mapping and analysis to flourish within law enforcement agencies. Software, training and resources are easily available to those interest in this use of GIS. Crime mapping is used by analysts in law enforcement agencies to map, visualize, and analyze crime incident patterns. Mapping crime, using Geographic Information Systems (GIS), allows crime analysts to identify crime hot spots, along with other trends and patterns (Morais, 2015). Therefore, Geographic Information Systems (GIS) and data mapping applications for crime mapping have become a necessary tool in law enforcement agencies worldwide, probably one of the most valuable tools available.

Satellite images can display important information about criminal activities happening around the nation and around our world. The added functionality of satellite imagery and GIS in computer mapping has increased the capabilities of crime fighting due to the efficiency and speed of the analysis.

GIS allows analysts to overlay other datasets such as census demographics, locations of stores, banks and schools, etc., to better understand the underlying causes of crime and help law enforcement administrators to devise strategies to deal with the problem. GIS is also useful for law enforcement operations, such as allocating police officers and dispatching to emergencies (Satellite Imaging Corporation, 2015). 
From the first stage of data collection to monitoring and modelling future scenarios to evaluate how, where and when to react, GIS include a vast number of applications such as (Ferreira, João and Martins, 2012):

- Monitoring citizen' alerts;

- Identifying areas of criminal activity - crime hotspots and other terminologies;

- Allocation of police infrastructures and policeman;

- Understanding in a more effective way crime distribution over space and time;

- Modelling and prediction of "critical areas" through pattern analysis;

- Monitoring results, resources and infrastructures;

- Application of Internet as a form of communication to better disseminate information about crime statistics, police initiatives and public safety programs, etc.

Nowadays, the boom of internet technologies, particularly web-based geographic information systems (GIS) technologies, is opening new opportunities for use of crime mapping to support crime prevention. Research indicates that the functions provided in web-based crime mapping are less than in most traditional crime mapping software. In conclusion, existing works of web-based crime mapping focus on supporting community policing rather than analytical functions such as pattern analysis and prediction.

This paper discussed the relevance of digital maps to crime management in Oshodi-Isolo Local Government Area (LGA) of Lagos State. Some basic policing strategies and the relationship between crime and environment were also discussed. Coordinates of police stations and crime spots were mapped and integrated with other spatially mapped objects such as street network in ArcGIS 10.1. Journey to Crime analysis, based Dijkstra's Shortest-Path Algorithm, was carried out to establish the relationship between crime spots and police stations. Closest Facility, Service Area and OD Cost Matrix were determined for the relationship.

\subsection{Materials and Methods}

\subsection{Study Area}

The study area is Oshodi-Isolo Local Government Area (LGA) of Lagos State (Fig. 1). Oshodi-Isolo is a Local Government Area (LGA) within Lagos State. The LGA is part of the Ikeja Division of Lagos State, Nigeria. At the 2006 Census it had a population of 621,509 people, and an area of 45 square kilometres.

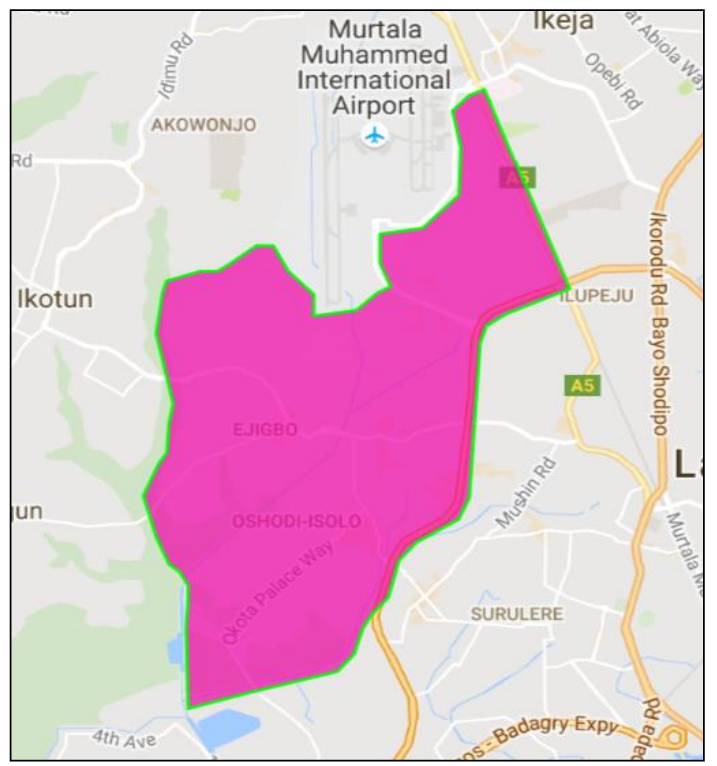

Figure 1: Map of Oshodi-Isolo Local Government Area

(City Population, 2016) 


\subsection{Some Basic Policing Strategies}

Ferreira, João and Martins (2012) described Community policing, problem-oriented policing and intelligence-led policing (ILP) as follows:

Community Policing (CP)- has its origins in the lack of interaction between the police and the community. According to the United States Community Policing Consortium, community policing is a joint collaboration between the police and the community that identifies and solves community problems. Within the concept, all the members of the community become active allies in the effort for the safety and quality of neighbourhood living. Mapping crime has been one of the most important trends and has grown considerably over the last few years. Crime data and modelling through Internet is a good example.

Problem-Oriented Policing (POP)- could be described as an approach or process within a police department or agency in which formal criminal justice theory, research methods, and comprehensive data collection and analysis procedures are used in systematic way to conduct in-depth examination of, developed, informed responses to, and evaluate crime and disorder problems" (Boba, 2003). So, this method is not only about creating maps, not only statistical analysis, not only identify patterns with models, it is "...examining the underlying conditions of both the simple and complex problems..." that modern police has to deal and respond adequately. Because of that, mapping has to overcome the simple process of draw a pin or flag map and evolve to an understanding of structural causes that lead to crime.

Intelligence-Led Policing (ILP)- The first stage in the development of ILP was in 1993 with an UK Audit Commission report titled "Helping with Enquires- Tackling Crime Effectively" (Audit Commission, 1993). Intelligence-Led Policing has as a specific "strategic, future-oriented and targeted approach to crime control (Maguire, 2000). The main focus is given to a prior analysis supporting decision-making processes. ILP is also more focused on individuals and groups of offenders and law enforcement solutions.

Ferreira, João and Martins (2012) concluded that, choosing between different policing approaches is probably far and beyond the control of most policemen as well as crime analysts. But however, it is crucial to perceive that all the three have different aims and objectives, and that in a real scenario it is probable that all of them could be used to obtain the best results when fighting a crime.

Predictive Policing- Similarly, Morais (2015) stated that most crime analysis produces maps and statistics for crimes that already have occurred. The Los Angeles Times has an article about predictive policing which looks to analyze when and where crimes are the mostly likely to occur.

Situational Crime Prevention- There are different ways of crime prevention. One of these is situational crime prevention, possibly the most spatial crime prevention concept and one that is a natural geographic corollary of rational choice theory. Situational crime prevention does not worry about why people commit crime, but concerns itself with preventing the opportunities for crime. It suggests a number of opportunity-reducing tactics that are crime-specific, that involve manipulating the immediate environment in a systematic and permanent way, and that are intended to increase the effort and risk while reducing the rewards that are perceived by a range of offenders (Clarke, 1992). Geographical Information Systems (GIS) lends itself to situational crime prevention studies due to the place-specific nature of the technique and the ability of GIS to deliver a spatial analysis.

In providing a way to influence someone not to commit a crime, three main strategies are suggested by situational crime prevention. First, it may be possible to increase the risks. By making the chance of capture much higher, offenders may make a rational choice to seek a less well-defended target. A second method of influencing the decision-making of offenders is to increase the effort required to commit a crime. Finally, it is possible to reduce the rewards of crime (Chainey and Ratcliffe, 2005). 


\subsection{GIS in Policing and Crime Prevention}

Crime mapping can play an important role in the policing and crime reduction process, from the first stage of data collection through to the monitoring and evaluation of any targeted response. It can also act as an important mechanism in a more pivotal preliminary stage, that of preventing crime by helping in the design of initiatives that are successful in tackling a crime problem. Many central processes to policing and crime reduction include the following application areas: (i) Recording and mapping police activity, crime reduction projects, calls for service and crime incidents; (ii) Supporting the briefing of operational police officers by identifying crimes that have recently occurred and predicting where crime may occur in the future; (iii) Identifying crime hotspots for targeting, deploying and allocating suitable crime reduction responses; (iv) Helping to effectively understand crime distribution, and to explore the mechanisms, dynamics and generators to criminal activity, through pattern analysis with other local data; (v) Monitoring the impact of crime reduction initiatives; and (vi) Using maps as a medium to communicate to the public crime statistics for their area and the initiatives that are being implemented to tackle crime problems (Chainey and Ratcliffe, 2005).

Also, crime analysts use crime mapping and analysis to help law enforcement management (e.g. the police chief) to make better decisions, target resources, and formulate strategies, as well as for tactical analysis (e.g. crime forecasting, geographic profiling). From a research and policy perspective, crime mapping is used to understand patterns of incarceration and recidivism, help target resources and programs, evaluate crime prevention or crime reduction programs (e.g. Project Safe Neighborhoods, Weed \& Seed and as proposed in Fixing Broken Windows [George, 1997; Catherine, 1996]), and further understanding of causes of crime.

\subsection{Relationship between Crime and Environment}

GIS is a powerful tool to assist in the management, integration and analysis of a disparate range of data (Burrough, 1998; Longley ci al., 2001). The need for integrating data on crime with other data on environmental, in form of layers, is very crucial for crime tracking, prevention and reduction. Data are essential to crime mapping. Without crime data and base maps, a GIS has no crime prevention or reduction application. (Chainey and Ratcliffe, 2005).

Crime has an inherent geographical quality. When a crime occurs, it happens at a place with a geographical location. For someone to have committed a crime they must have also come from a place (such as their home, work or school). This place could be the same location where the crime was committed or is often close to where the crime was perpetrated. Crime records/data must contains details on the crime (such as type of crime, people affected by the crime, etc.) and the geographic locational information that are geocoded on a map. Geographic references (such as postal address, postcodes and other generalized address data) must accompany crime data. This address is then automatically entered into the crime record with its geographic coordinates (Frisbie et al., 1977; Rossmo, 2000; Wiles and Costello, 2000; Chainey and Ratcliffe, 2005; IACA, 2012).

\subsection{Shortest-Path Determination using Dijkstra's Algorithm}

In journey to crime, offenders restrict themselves in how they move around space and they tend to follow basic rules in regard to their movement. The behavioural axiom of the least effort principle suggests that people will usually exert the minimum effort possible to complete their tasks. This therefore implies that there is a decay in the frequency of activity as distance increases (Fig. 2). In other words, this theory means that offenders tend to travel short distances on average to commit their crimes. The routine activities that people take (be they offenders or potential victims) also need to be considered when understanding the routes and movements that offenders take to commit crime. Appreciating the likely distance that an offender may travel to commit their crime is an important component in tactical and investigative analysis (Chainey and Ratcliffe, 2005), Figure 2 shows distance decay as a simple linear relationship. This works as a basic theoretical concept; however, the reality of distance decay is often more complex. 


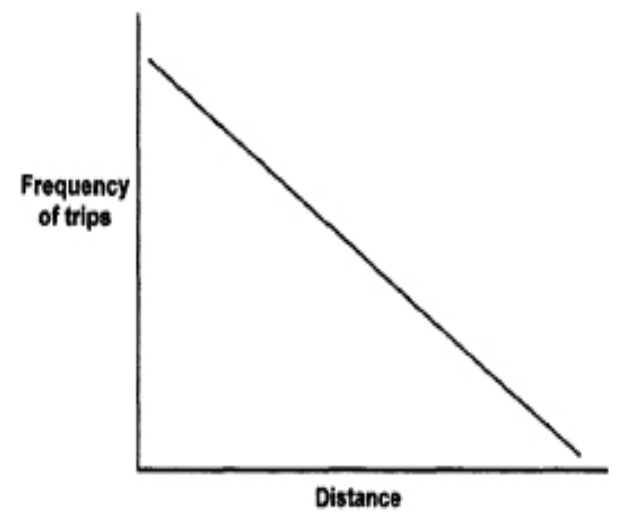

Figure 2: The Distance Decay function.

Real distance decay functions can vary in shape and linear orientation (e.g. the decay could be exponential) in response to different types of movement behaviour (Longley Ct al., 2001). For examples shopping trips can be divided into two general categories.

1. Convenience shopping is characterized by a high frequency of short trips.

2. Comparison shopping occurs when buyers are seeking more expensive items (Harries, 1999).

These differences in distance decay could also be applied to crime where opportunity-based criminal behaviour may be more frequent over shorter distances, compared to organized and planned criminal behaviour that may require travelling further distances to complete criminal acts.

For a criminal, shortest-path/route to where to commit crime and shortest-path/route for safe escape to their abode or hideout are very paramount to his nefarious activities. Similarly, for a policeman to get to a crime scene or a criminal hideout, shortest-path/route(s) is very necessary for an efficient crime prevention or arrest. Therefore, shortest-path algorithm is very germane in crime mapping and analysis.

In graph theory, finding a path between two nodes (vertices) in a graph such that the sum of the weights of its constituent edges is minimized is crucial. The nodes represent the locations (such as origin and destination of a criminal or a policeman), while the road segments represent the edges and the weights represent the travel time on that segment. The travel time, which is a function of shortest-path, in this research is constrained based on distance between origin and destination.

According to Goodrich and Tamassia (2002), for Single-Source Shortest Paths, let $G$ be a weighted graph. The length (or weight) of a path $P$ is the sum of the weights of the edges of $P$. That is, if $P$ consists of edges $e_{0}, e_{1}, \ldots \ldots ., e_{k-1}$ then the length of $\mathrm{P}$, denoted by $w(P)$, is defined as:

where:

$$
w(P)=\sum_{i=0}^{k-1} w\left(e_{i}\right)
$$

$$
\begin{aligned}
& w(P) \text { - the length (or weight) of a path } P . \\
& w\left(e_{i}\right) \text { - weights of the edges of } P \\
& e_{i} \text { - edges } e_{0}, e_{1}, \ldots \ldots ., e_{k-1}
\end{aligned}
$$

The distance from a vertex $v$ to a vertex $u$ in $G$, denoted $d(v, u)$, is the length of a minimum length path (also called shortest path) from $v$ to $u$, if such a path exists. Dijkstra's algorithm (Pryor, Kinahan and Chinneck, 2007) in Figures $3 a-3 c$ illustrates the solution to the shortest path problem from the origin $O$ to the destination $T$. Distances (weights) between nodes are labelled on the edges. 


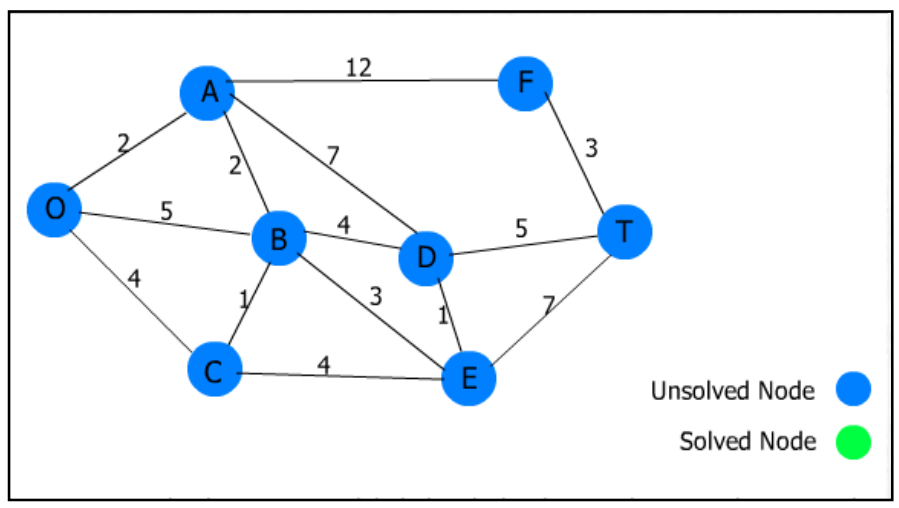

Figure 3a: Shortest Path from $O$ to $T$ to be determined.
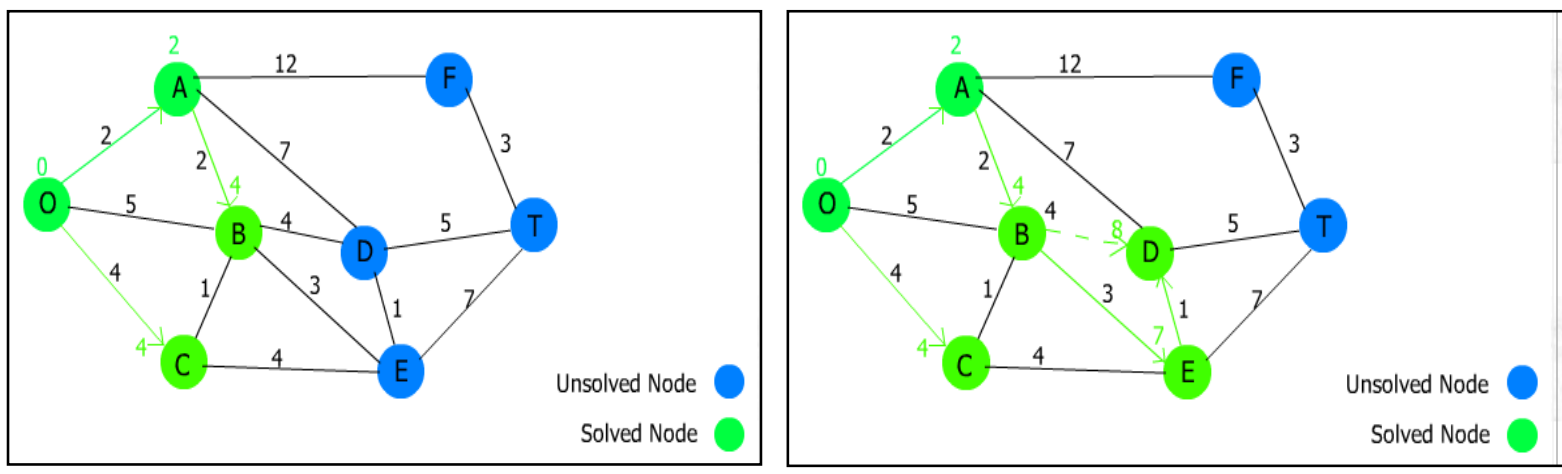

Figure 3b: Determining Shortest Path from $O$ to $T$.

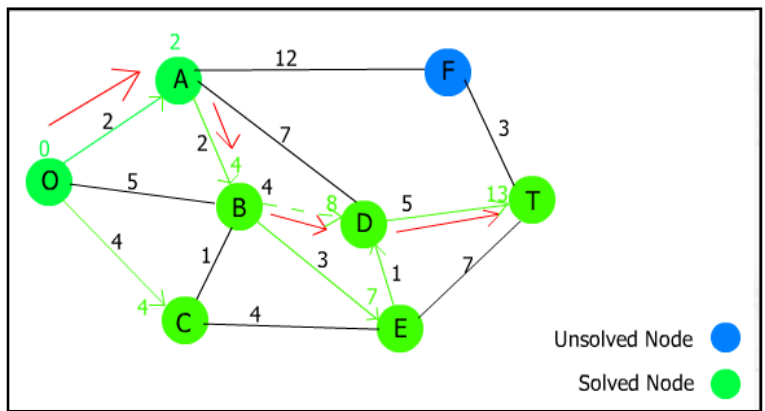

(i) The First Shortest Routes: $O-A-B-D-T$.

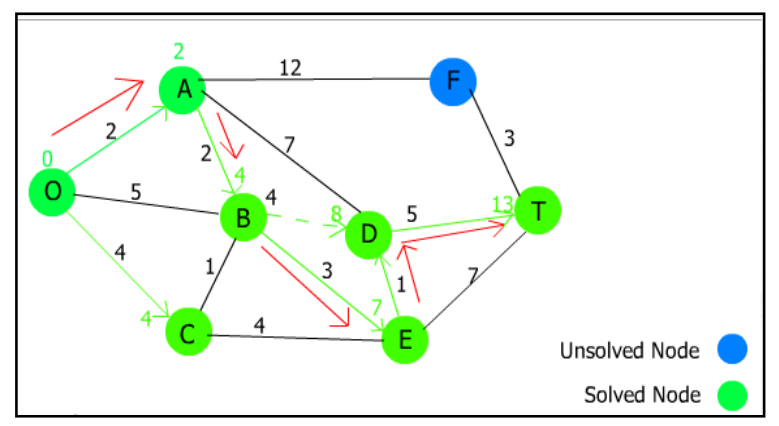

(ii) The Second Shortest Routes: $O-A-B-E-D-T$

Figure 3c: Determining Shortest Path from $O$ to $T$ (Pryor, Kinahan and Chinneck, 2007)

However, for All-Pairs Shortest Paths, to compute the shortest path distance between every pair of vertices in a directed graph $\vec{G}$ with $n$ vertices and $m$ edges with $\vec{G}$ having no negative-weight edges, the Dijkstra's algorithm can also be used. For this work, since a criminal or a policeman is interested in allpairs shortest path distances and then take decision, a dynamic programming algorithm to compute allpairs shortest path distances in a digraph without negative cycles is described below.

\section{A Dynamic Programming Shortest Path Algorithm}

Let $\vec{G}$ be a given weighted directed graph. The vertices of $\vec{G}$ is numbered as $\left(v_{1}, v_{2}, \ldots . ., v_{n}\right)$. As in dynamic programming algorithm, the key construct in the algorithm is to define a parameterized cost function that is easy to compute and also allows us to ultimately compute a final solution. In this case, we use the Cost function, $D_{i, j}^{k}$, which is defined as the distance from $v_{i}$ to $v_{j}$ using only intermediate vertices in the set $\left\{v_{1}, v_{2}, \ldots \ldots, v_{k}\right\}$. Initially,

$$
D_{i, j}^{0}=\left\{\begin{array}{cc}
0 & \text { if } i=j \\
W\left(\left(v_{i}, v_{j}\right)\right) & \text { if }\left(v_{i}, v_{j}\right) \text { is an edge in } \vec{G} \\
+\infty & \text { otherwise }
\end{array}\right.
$$


Given this parameterized cost function $D_{i, j}^{k}$, and its initial value $D_{i, j}^{0}$, we can then easily define the value for an arbitrary $k>0$ using Floyd-Warshall algorithm as:

$$
D_{i, j}^{k}=\min \left\{D_{i, j}^{k-1}, D_{i, k}^{k-1}+D_{k, j}^{k-1}\right\}
$$

In other words, the cost for going from $v_{i}$ to $v_{j}$ using the vertices numbered 1 through $k$ is equal to the shortest of two possible paths. The first path is simply the shortest path from $v_{i}$ to $v_{j}$ using the vertices numbered 1 through $k-1$. The second path is the sum of the costs of the shortest path from $v_{i}$ to $v_{k}$ using vertices numbered 1 through $k-1$ and the shortest path from $v_{k}$ to $v_{j}$ using vertices numbered 1 through $k$-1. Moreover, there is no other shorter path from $v_{i}$ to $v_{j}$ using vertices of $\left\{v_{1}, v_{2}, \ldots \ldots, v_{k}\right\}$ than these two. If there was such a shorter path and it excluded $v_{k}$, then it would violate the definition of $D_{i, j}^{k-1}$, and if there was such a shorter path and it included $v_{k}$, then it would violate the definition of $D_{i, k}^{k-1}$ or $D_{k, j}^{k-1}$. In fact, note that this argument still holds even if there are negative cost edges in $\vec{G}$, just so long as there are no negative cost cycles. The cost functions in practical travel time should include: congestions cost, signalized and non-signalized intersections and wrong driving.

Dijkstra's algorithm has had its wide applications in the following transportation challenges (Esri, 19952012):

$>$ Route- uses the well-known Dijkstra's algorithm quickest/shortest route with travel time to a crime location as impedance.

> Closest Facility- uses a multiple-origin, multiple-destination algorithm based on Dijkstra's algorithm, and has options to only compute the shortest paths if they are within a specified cutoff or to solve for a fixed number of closest facilities.

$>$ Service Area- is also based on Dijkstra's algorithm to traverse the network. Its goal is to return a subset of connected edge features such that they are within the specified network distance or cost cutoff; in addition, it can return the lines categorized by a set of break values that an edge may fall within.

$>$ OD Cost Matrix- uses a multiple-origin, multiple-destination algorithm based on Dijkstra's algorithm also. It has options to only compute the shortest paths if they are within a specified cutoff or to solve for a fixed number of closest destinations. The OD Cost Matrix solver does not compute the shape of the resulting shortest path for less overhead and faster performance.

All these were carried out in this work. Being in a GIS environment, Start Time, Time of Day, Specific Date, Day of Week and others can be used in the O-D matrix modelling, while restrictions like "No UTurn", and so on can also be modelled in the analysis.

\subsection{Data Acquisition}

The steps involved in the acquisition of data include:

(i) Crime locations were identified through police records and public interviews; (ii) Questionnaires were administered to the residents in the study area as well as oral interviews conducted. The questionnaires were randomly distributed to residents in these identified locations; (iii) Geographic coordinates of these identified spots were obtained using handheld GPS.

In general, questions were asked both on spatial and attribute data before mapping was carried out. Questions like, crime location, time and date of crime, people involved, suspects' description, government agency presence or intervention and action by local security. Cross-examine information from different sources to confirm data reliability or credibility was also ensured.

\subsection{Data Processing}

In this research, street network in the study area represents the edges (i.e. routes from the criminal hideout or police station to the crime scene), while the crime locations; such as burglary, pick-pocketing, rape, armed robbery, and others; are represented by nodes (points). Topology was built to ensure connectivity and establish relationships between features on the map. Spatial data were plotted in 
ArcGIS 10.1 environment with the attribute table showing all the collected descriptions of the crime location, crime committed and time component.

The attribute data of all crimes mapped were collected and scrutinized for accuracy before database was created. The scrubbing/cleaning procedure (which includes multiple address, abbreviations, and incomplete address) was carried out before proper address geocoding took place. The mapped crime points and police stations were created as thematic maps for easy police-crime spot matching. That is, which police station is responsible for crime in a particular location?

Spatial locations where the number of crimes are higher than other areas in a neighborhood were identified i.e. the Hotspots. A hotspot represents an area of high crime concentration, relative to the distribution of crime across the whole region of interest (Chainey and Ratcliffe, 2005).

\subsection{Results and Discussion}

\subsection{Results}

Apart from mapping the crime spots, the police stations; queries on Route (i.e. Shortest-Path), Closest Facility, OD Cost Matrix, Service Area were all carried out. Results obtained from the study area are shown below (Figures. 4a-5c).

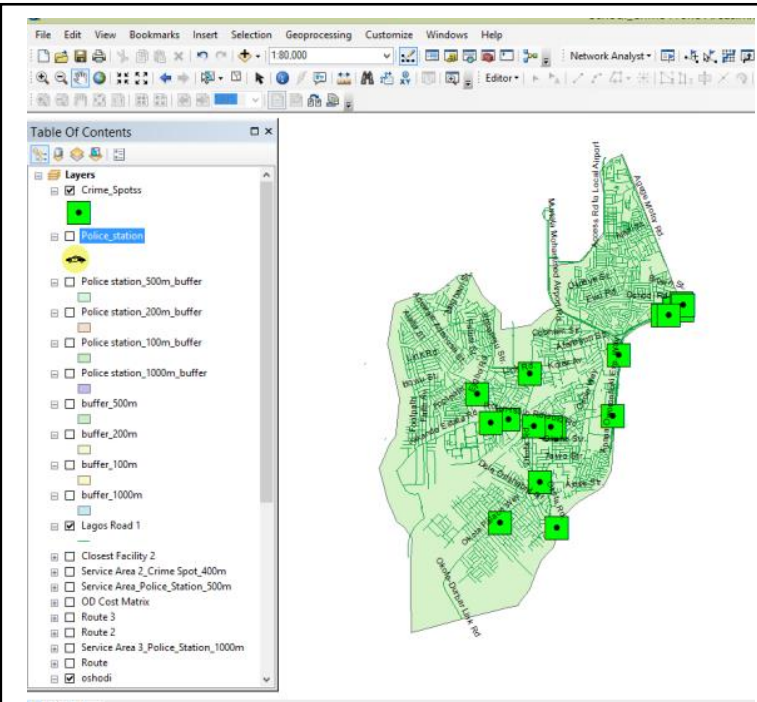

Figure 4a: Point Map showing some Crime Spots.

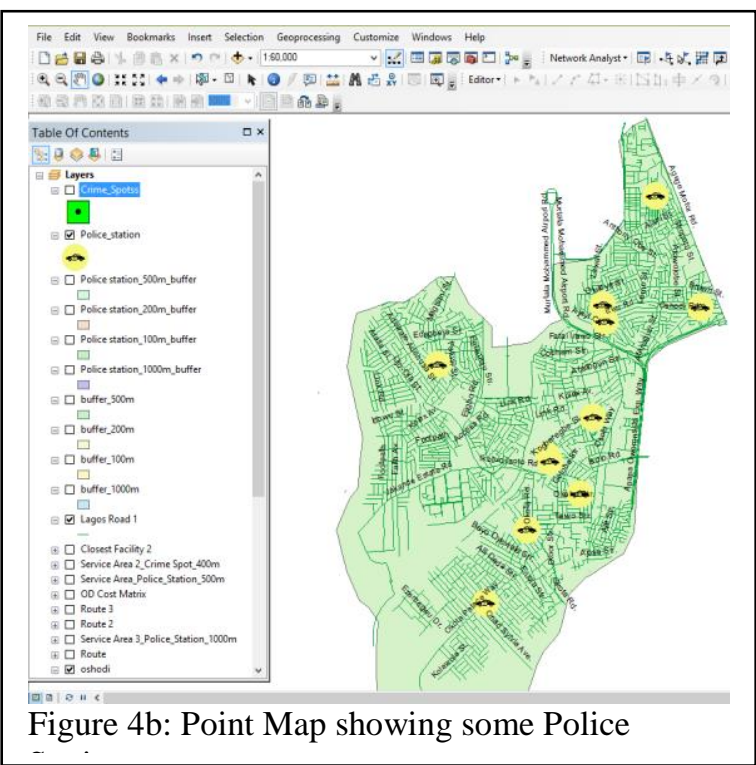



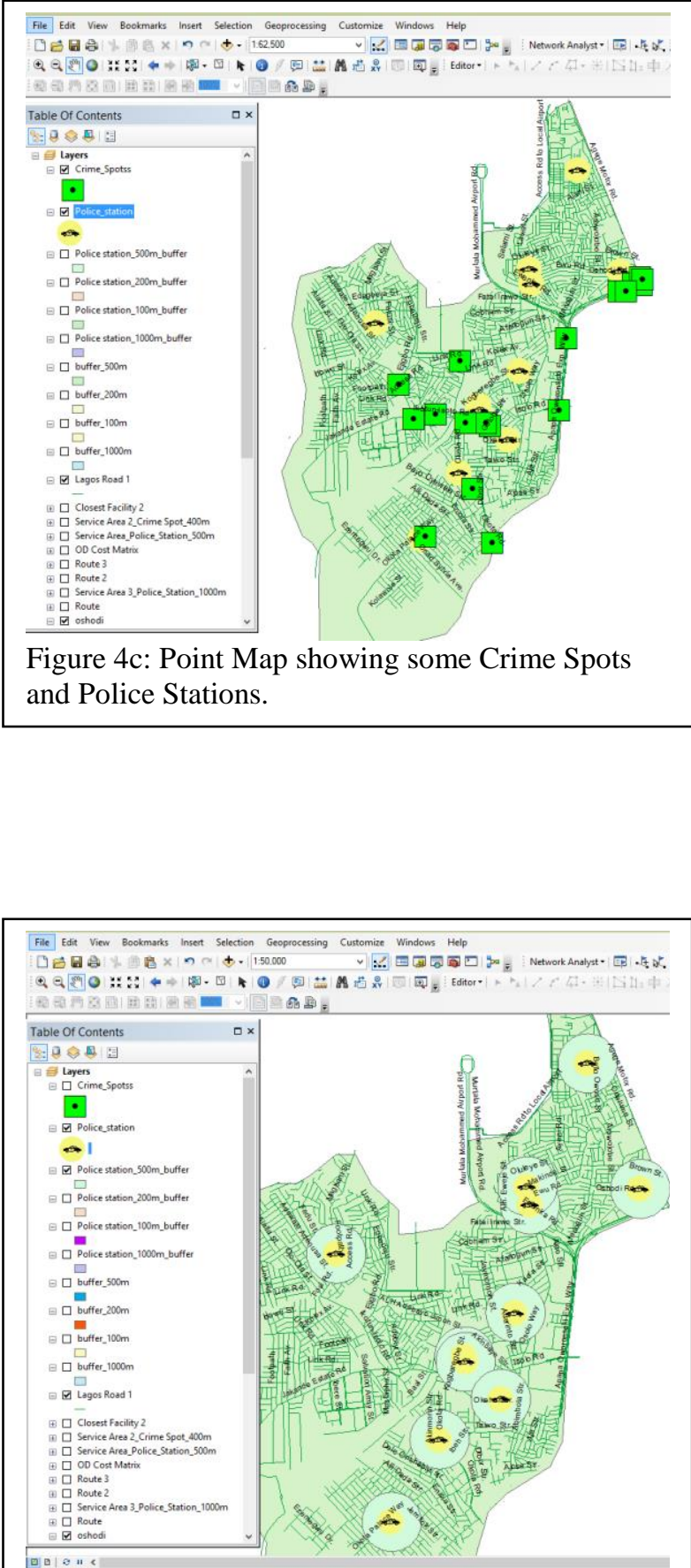

Figure 4e: Point Map showing 500m-Buffers from some Police Stations.
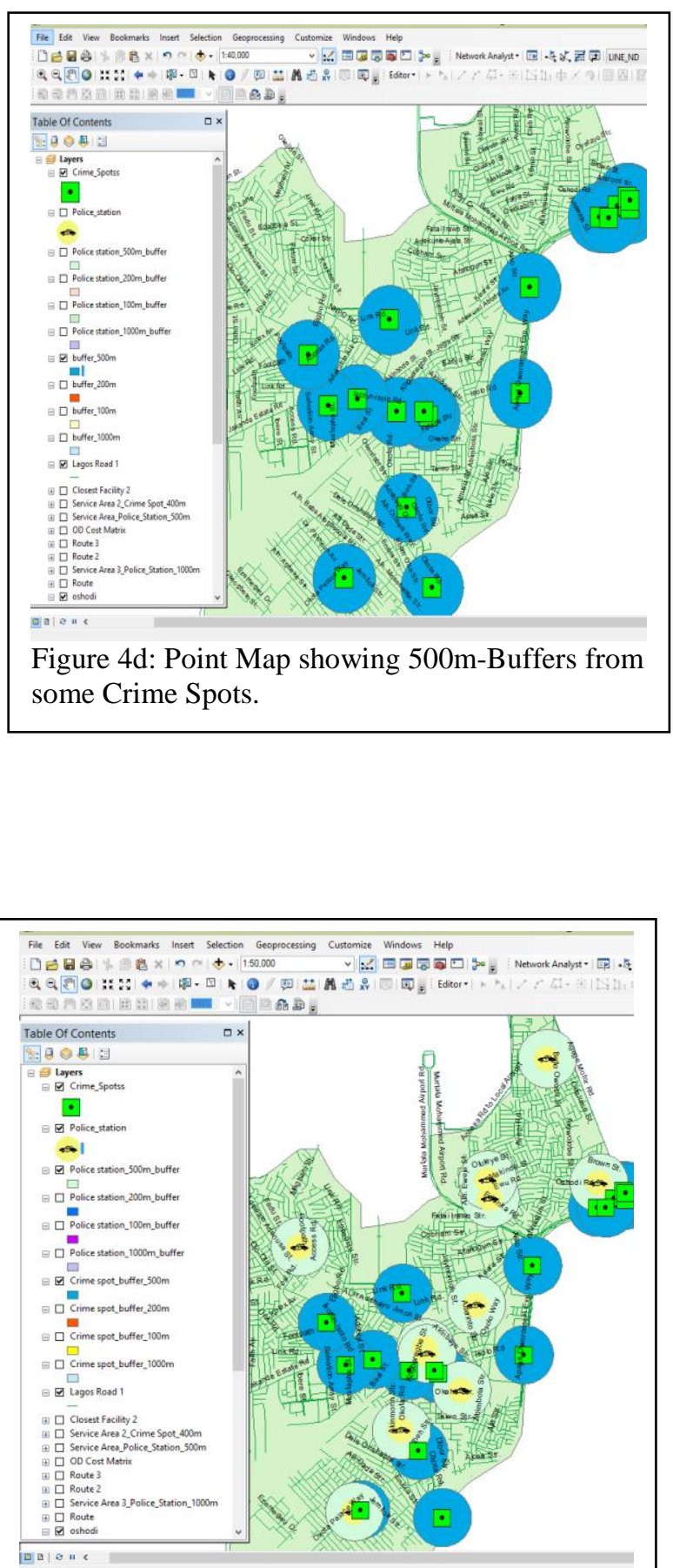

Figure 4f: Point Map showing 500m-Buffers from some Police Stations and Crime Spots. 


\section{$\underline{\text { Shortest-Path Analysis }}$}

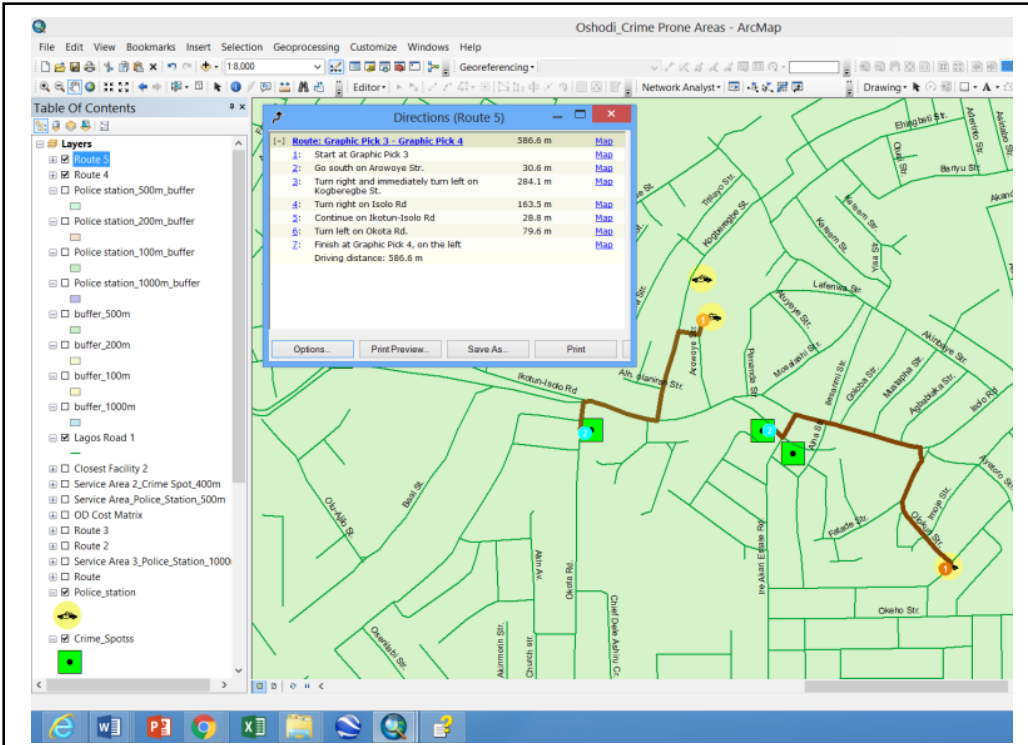

Figure 5a: Map showing Shortest-Paths from two Police Stations to two different Crime Spots, while the Direction from The Nigerian Police Post (Panada Street) to a Crime Spot at Parko Round About, Isolo where Robbery and Hijacking do take place is shown in the inset box.

\section{Closest Facility-Model}

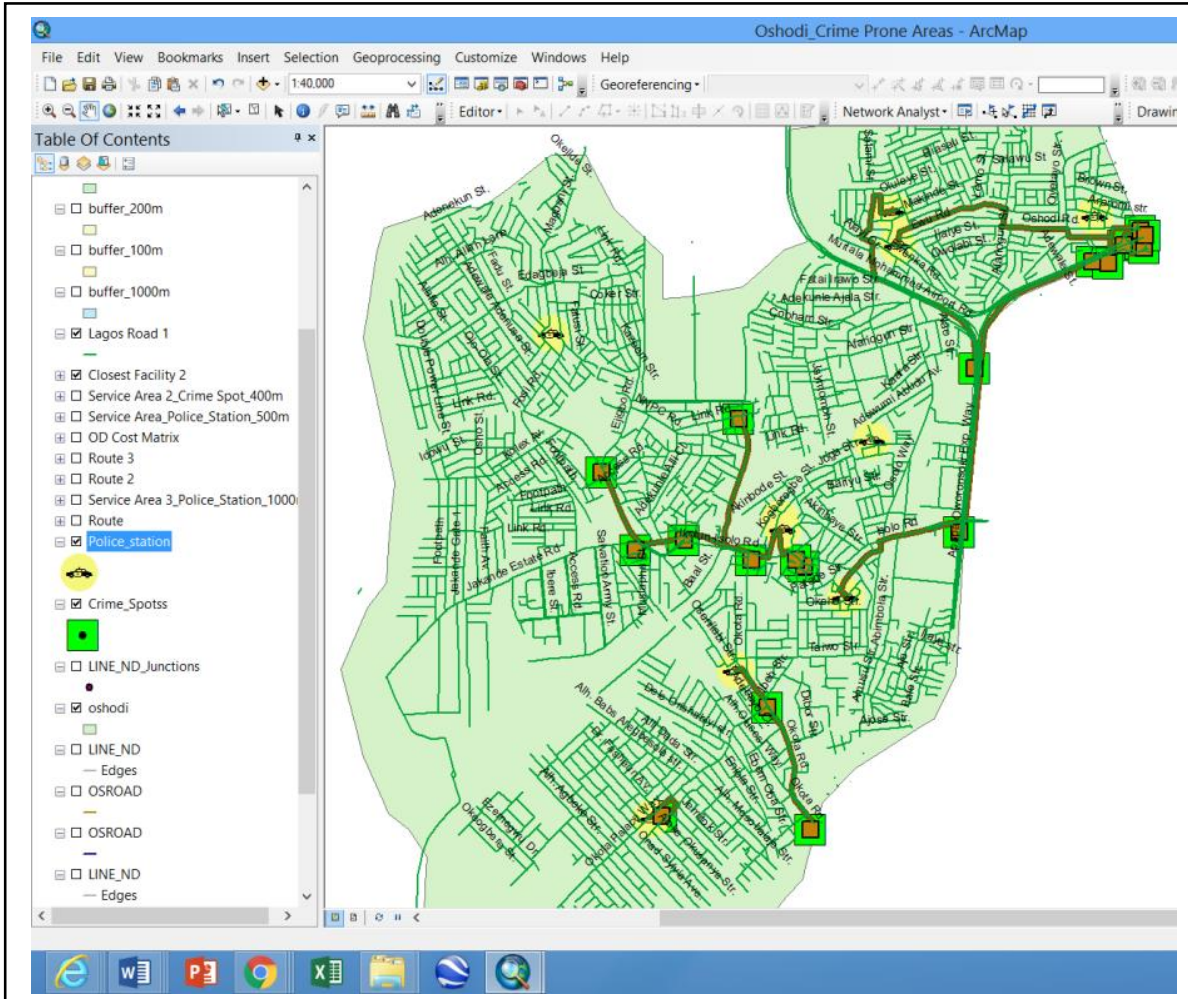

Figure 5b: Map showing Police Stations Closest to Crime Spots.

From Figure 5b, some police stations are not close to any known crime spot. Meaning that criminals can be having a "field day". 
$\underline{\text { Service Area-Model }}$

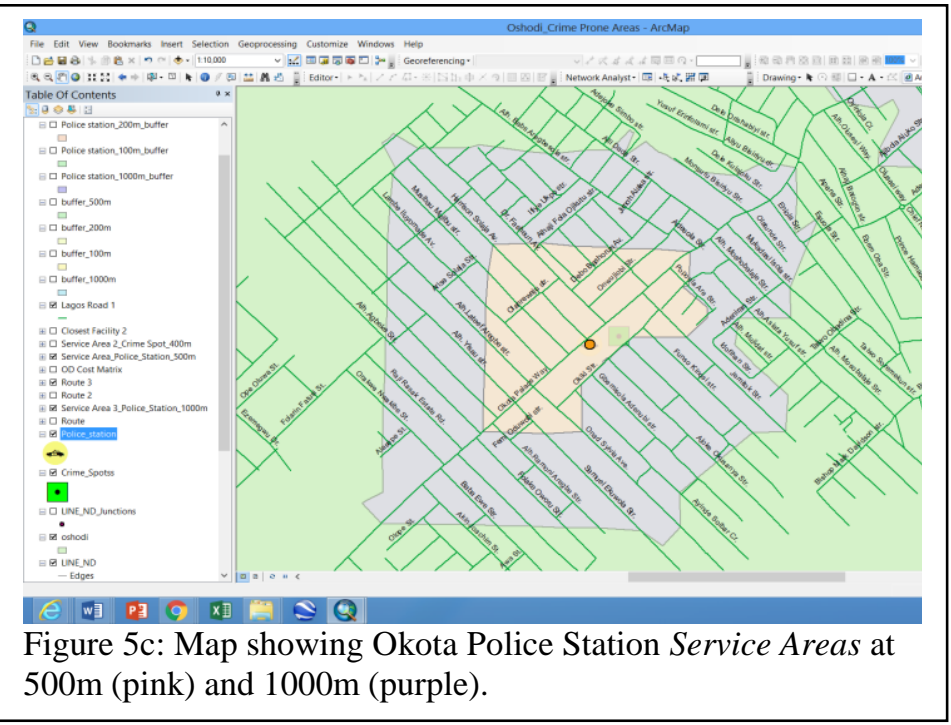

Origin-Destination Matrix-Model

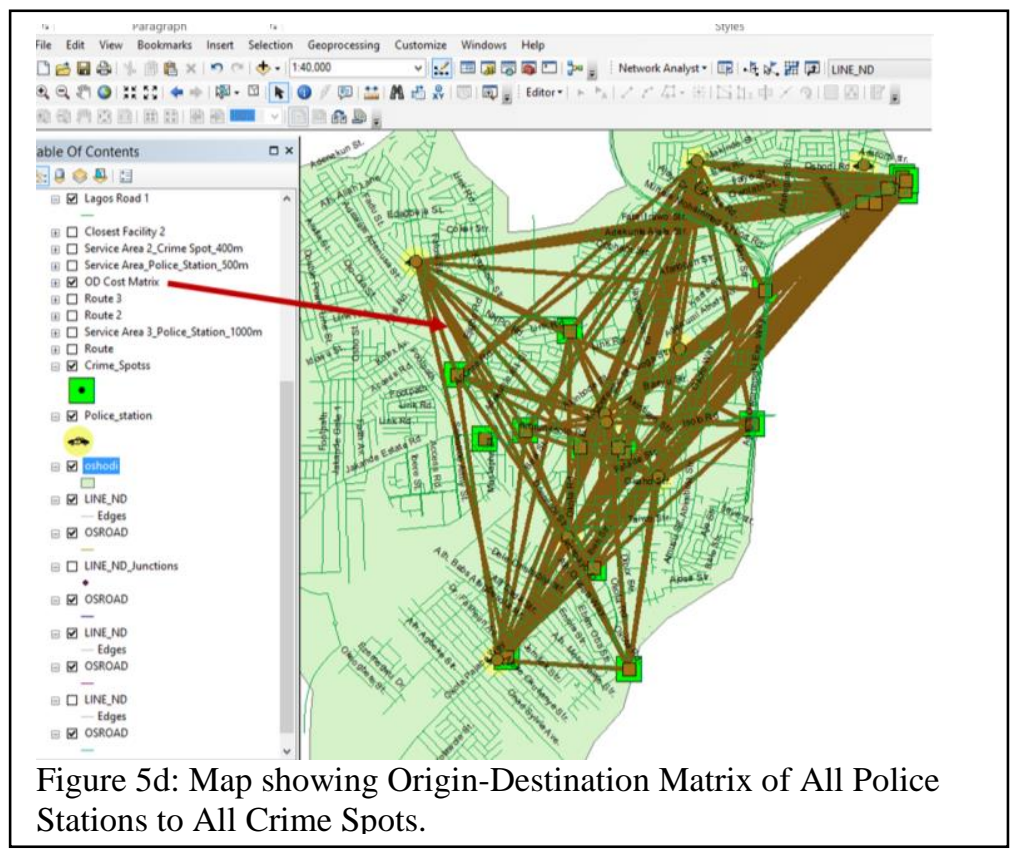

\subsection{Discussion}

For Best Route- in Figure 5a, distance as an impedance, the quickest path is shown in blue and has a total length of 4.5 miles, which takes 8 minutes to traverse. Also, directions with turn-by-turn maps were generated.

For Closest Facility- Finding the closest police stations (facilities) to crime spots (incidences) or sometimes the closest hospitals to crime spots. The best route and direction from the closest police station to a crime spot and the travel cost (time) can be displayed graphically, as in Figure 5a. Similarly, one can query for a closest police station (facility) or hospitals within $500 \mathrm{~m}$ or $1000 \mathrm{~m}$ buffer zones (Fig.4f) with a 15 minutes' drive time to a crime spot. Any police stations that take longer than 15 minutes to reach the crime spot will not be included in the result.

Service Areas- Alternatively, in terms of crime management, Coverage Area (Service Area) is the area of jurisdiction that each police station/post should cover. A Service Area of 500m from each police station was first used [pink] (Fig. 5c). That is, this includes all the streets that are within $500 \mathrm{~m}$. Where government resources cannot provide enough coverage at this range, a $1000 \mathrm{~m}(1 \mathrm{~km})$ in purple Service 
Area can be adopted in order to have a wider coverage. Multiple concentric Service Areas show how accessibility changes with an increase in impedance. It can be used, for example, to show how many police stations are within 5-, 10-, and 15-minute drive times to crime spots.

Service Area functionality (Figure 5c), can be used by the Police Authority to indicate area of jurisdiction that a police station with a Divisional Police Officer (DPO) is to cover. That is, the police station will be responsible for managing crimes within that service area or zone.

Origin-Destination Matrix- Figure 5d has a Many-Many Relationship i.e. any police station can be called upon to assist in curbing crime at any location withing the Local Government Area. This is very necessary in case of need for reinforcement by the police to overpower armed robbery gang and other notorious gangs.

In all, Origin $($ Police stations $)=11$; Destination $($ Crime spots $)=19$; Both $=30$ i.e. 11 police stations are to cover 19 identified crime spots.

\subsection{Conclusion}

Overlaying other datasets such as census demography, school locations, local government boundary, with crime data in a Geographic Information Systems (GIS) environment is very crucial in crime management. Solutions can be proffered to various security challenges at local, regional, national and international levels using the integration of GIS and other information technologies. By mapping and analyzing historical crime data, will assist in identifying crime hot spots (such as Oshodi Bus stop), along with other trends and patterns. "what-if" scenarios can be painted and analyzed in crime detection and prevention.

Crime and police data were collected, mapped and analysed. Distance Decay function that was based on Dijkstra's Shortest-path algorithm was used to understand journeys made by criminals from their hideouts to the crime spots.

Until now, most Divisional Police stations and State Police Commissioner Offices in Nigeria are not well equipped with digital crime mapping technology. Similarly, the use of Navigators, equipped with up-to-date street map, that will guide a police officer/team to crime locations or criminal hideouts are still lacking in police vehicles. The dearth of this very important technology has hampered the efficiency and effectiveness of our security agencies.

\section{References}

Audit Commission (1993), Helping With Enquiries: Tackling Crime Effectively, London, HMSO. In Ferreira, J, João, P and Martins, J. (2012). "GIS for Crime Analysis - Geography for Predictive Models" The Electronic Journal Information Systems Evaluation, Volume 15 Issue 1 2012, ISSN 1566-6379 36 CAcademic Publishing International Ltd, p. 36-49. Available at: www.ejise.com.

Boba, R. (2003), Problem analysis in policing. Washington, DC: Police Foundation. In Ferreira, J, João, P and Martins, J. (2012). "GIS for Crime Analysis - Geography for Predictive Models" The Electronic Journal Information Systems Evaluation, Volume 15 Issue 1 2012, ISSN 1566-6379 36 @Academic Publishing International Ltd, p. 36 - 49. Available at www.ejise.com.

Burrough, P.A. (1998). Principles of Geographical Information Systems for Land Resources Assessment. Oxford, Oxford University Press.

Chainey S. and Ratcliffe J. (2005). GIS and crime mapping. John Wiley \& Sons Ltd, The Atrium, Southern Gate, Chichester, West Sussex P019 8SQ, England. 
City Population (2016). OSHODI-ISOLO (Local Government Area, Metro Lagos). [online]. Available at: https://www.citypopulation.de/php/nigeria-metrolagos.php?cid=NGA025018.

Clarke, R.V. (1992). Situational Crime Prevention: Successful Case Studies (p. 286). Albany, NY: Harrow and Heston. In Chainey S. and Ratcliffe J. (2005). GIS and crime mapping. John Wiley \& Sons Ltd, The Atrium, Southern Gate, Chichester, West Sussex P019 8SQ, England.

Burrough, P.A. (1998). Principles of Geographical Information Systems for Land Resources Assessment. Oxford: Oxford University Press. In Chainey S. and Ratcliffe J. (2005). GIS and crime mapping. John Wiley \& Sons Ltd, The Atrium, Southern Gate, Chichester, West Sussex P019 8SQ, England.

Esri, ArcGIS 10.1 Software Online Help, 1995-2012.

Ferreira, J, João, P and Martins, J. (2012). "GIS for Crime Analysis - Geography for Predictive Models" The Electronic Journal Information Systems Evaluation, Volume 15 Issue 1 2012, ISSN 1566-6379 36 (C)Academic Publishing International Ltd, p. 36 -49. Available at: www.ejise.com.

Frisbie, D.W., Fishbine, G., Hintz, R., Joelson, M. and Nutter, J.B. (1977). Crime in Minneapolis: Proposals for prevention. St Paul, MN: Community Crime Prevention Project, Governor's Commission on Crime Prevention and Control.

George K. (1997); Catherine C. [1996]. Fixing Broken Windows: Restoring Order and Reducing Crime in Our Communities. New York: Simon \& Schuster. ISBN 0-684-83738-2. In Wikipedia ${ }^{1}$, the free encyclopaedia (Saved, 2015). Crime mapping. Available at: http://en.wikipedia.org/wiki/Crime_mapping; http://en.wikipedia.org/wiki/Crime_mapping.

Goodrich M.T. and Tamassia R. (2002). “Algorithm Design Foundations, Analysis, and Internet Examples. John Wiley \& Sons, Inc. [online]. Available at: http://www.wiley.com/college/goodrich/.

Harries, K. (1999). Mapping Crime: Principles and Practice. Washington, DC: United States Department of Justice. [online] Available at: http://www.ojp.usdoj.gov/nij/maps/.

International Association of Crime Analysts (IACA) (2012). GIS Software Requirements for Crime Analysis: Standards, Methods, \& Technology (SMT) Committee White Paper 2012-01 June 2012.

Longley, P., Goodchild, M., Maguire, D. and Rhind, D. (2001). Geographic Information Systems and Science. Chichester: John Wiley \& Sons. In Chainey S. and Ratcliffe J. (2005). GIS and crime mapping. John Wiley \& Sons Ltd, The Atrium, Southern Gate, Chichester, West Sussex P019 8SQ, England.

Maguire, M. (2000), Policing by risks and targets: Some dimensions and implications of intelligenceled crime control", Policing and Society, p.3. In Ferreira, J, João, P and Martins, J. (2012). "GIS for Crime Analysis - Geography for Predictive Models" The Electronic Journal Information Systems Evaluation, Volume 15 Issue 1 2012, ISSN 1566-6379 36 @Academic Publishing International Ltd, p. 36 -49. Available at www.ejise.com.

Morais C. D. (2015). GIS Learning: Crime Mapping and Analysis. [online]. Available at: http://www.gislounge.com/gis-101/.

Pryor J., Kinahan K. and Chinneck J. (2007). Example Networks1: Dijkstra's Algorithm for Shortest Route Problems. Carleton University. [online] Available at: http://optlabserver.sce.carleton.ca/POAnimations2007/DijkstrasAlgo.html.

Rossmo, K. (2000). Geographic Profiling. Boca Raton, Florida: CRC Press. 
Satellite Imaging Corporation (2015). Crime Mapping. Available at: http://www.satimagingcorp.com/ Copyright (C) 2001-2017.

Wiles, P. and Costello, A. (2000). The road to nowhere: The evidence for traveling criminals. [online] Home Office Research Study 207, Research, Development and Statistics Directorate, Home Office. Available at: http://www.homeoffice.gov.uk/rds/pdfs/hors207.pdf. 